\title{
The Study of Warehousing Slotting Optimization Based on the Improved Adaptive Genetic Algorithm
}

\author{
Lvqing Yang ${ }^{1, a}$, Wei-wei LIU ${ }^{2, b^{*}}$, Youjing Bai ${ }^{1, c}$ \\ ${ }^{1}$ Software School,Xiamen University, Xiamen 361005,China \\ ${ }^{2}$ Faculty of Informatics, Fujian Vocational College of Agriculture, Fuzhou, Fujian 350001 , \\ China

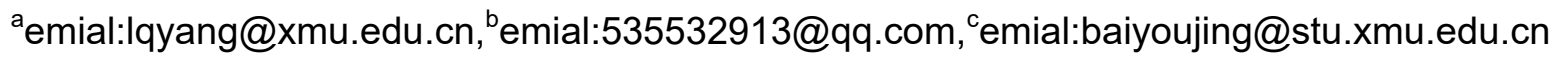 \\ *Corresponding author
}

Keywords: Storage efficiency, Slotting optimization, The improved adaptive genetic algorithm, Multi- objective optimization.

\begin{abstract}
Based on the linear adaptive genetic algorithms presented by Srinivas M, this paper improves the algorithm from the representation of the population diversity, proposing a new adaptive genetic algorithm; According to the basic principle of warehousing and the status of inventory, we summarize the storage location selection as a problem of multi-objective optimization, and establish a corresponding mathematical model; Using the improved algorithm to solve the storage location model indicates that the algorithm can better work out the problem of warehousing and the best location, which proves the validity of the algorithm .
\end{abstract}

\section{Introduction}

Slotting optimization means dynamically reallocating the goods location in the warehouse based on the current location of the goods and on the changes of SKU (Stock Keeping Unit) and unexpected changing factors, so as to ensure the goods distribution in a reasonable condition, and to achieve the goal of improving picking efficiency and reducing the operation cost of warehouse. The SKU factors are the internal cause of the slotting optimization, referring to the characteristics of goods shelves and goods. The unexpected changes are the external factors, referring to the mobility of goods.

Slotting optimization can present the gold region to the goods picked up in high frequency, thus to achieve the maximum efficiency and the minimum picking cost. And it can also facilitate the replenishment, balance the workload of the work area, provide effective support for warehouse management, and meanwhile improve the accuracy of picking, etc. At present many logistics management companies have released lots of slotting optimization software, which can be divided 
into two kinds: one is bound to WMS, while the other is separated from WMS and suitable for small- scale warehouses. The considered principles of location allocation generally include [1]: shelf load equality, top light Lower weight; speed up the turnover, first in first out; improve reliability, sub roadway storage; increase efficiency, the nearest out of storage, product correlation and so on. The storage location allocation and implementation strategies include [2]: classified storage strategy; improved advanced first out strategy; random storage strategy; cargo relevance strategy; shared memory strategy and so on.

\section{Storage location allocation model}

The storage location optimization can be described as follows:

Known: see the data in Table 1 and the related data of goods put in storage

Constraints: (1)the principles of location allocation,

(2)the storage condition of the current warehouse

Target: optimize the storage space; improve the storage utilization and storage efficiency

The storage allocation model in this paper is composed of five sub models, the value of which is needed to be considered when selecting the optimal location for the storage of goods. According to the research results of Yunwei Shang et al[3], Lingying Zheng et $\mathrm{al}^{[4]}$, Shiwen Tai et $\mathrm{al}^{[5]}$, the location allocation can be concluded as a problem of multi-objective optimization. The model of the storage location allocation is shown in Formula (1), and the related variables are described in Table 1:

$$
\begin{aligned}
& \left\{\begin{array}{l}
\min f_{1}=\left|x_{\text {pos }}-a\right|+\left|y_{\text {pos }}-b\right| \\
\min f_{2}=\sqrt{\left(x_{\text {pos }}-x_{0}\right)^{2}+\left(y_{\text {pos }}-y_{0}\right)^{2}}
\end{array}\right. \\
& \min f_{3}=\sqrt{\left(x_{\text {pos }}-x_{k}\right)^{2}+\left(y_{\text {pos }}-y_{k}\right)^{2}}
\end{aligned}
$$

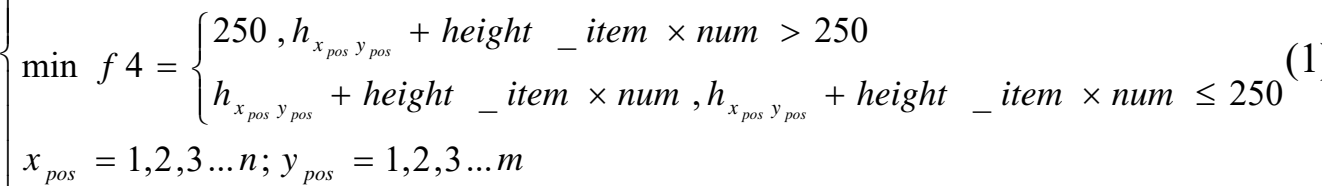

$$
\begin{aligned}
& \min f_{5}=\left\{\begin{array}{l}
1, \text { time }-i t e m<t_{x_{p o s} y_{p o s}} \\
3, \text { time_item } \geq t_{x_{p o s} y_{p o s}}
\end{array}, x_{p o s}=1,2,3 \ldots n ; y_{p o s}=1,2,3 \ldots \mathrm{m}\right.
\end{aligned}
$$


Table 1 Dependent variable description

\begin{tabular}{l|l}
\hline $\begin{array}{l}\text { variable } \\
\text { symbol }\end{array}$ & variable description \\
\hline $\begin{array}{l}(\mathrm{a}, \mathrm{b}) \\
\left(\mathrm{x}_{0}, \mathrm{y}_{0}\right) \\
\left(x_{k}, y_{k}\right) \\
\left(x_{\text {pos }}, y_{\text {pos }}\right)\end{array}$ & $\begin{array}{l}\text { XY coordinates of the inlet port } \\
\text { the mean value coordinates of the same order goods location } \\
\text { the mean value coordinates of the same order location } \\
\text { XY coordinates of the optional location } \\
\text { the height of the pile which uses }\left(x_{p o s}, y_{\text {pos }}\right) \text { as the } \\
t_{\text {xposypo }}\end{array}$ \\
$\begin{array}{l}\text { coordinates, unit centimeter } \\
\text { the earliest delivery time of the goods in the XY coordinates } \\
\text { pile } \\
\text { the height of each location ,unit centimeter } \\
\text { the storage time of the goods to be put in storage at present } \\
\text { time_item } \\
\text { the maximum XY coordinates of the warehouse location } \\
250\end{array}$ & $\begin{array}{l}\text { pile height limit, unit centimeter. Reference to the height } \\
\text { requirements of the enterprise }\end{array}$ \\
\hline
\end{tabular}

\section{Improved adaptive genetic algorithm}

The genetic algorithm (GA) is a kind of random search algorithm based on the biological evolution mechanism, which can effectively carry out the global search in the sense of probability [12]. Currently the common adaptive genetic algorithms include: linear adaptive genetic algorithm, cosine adaptive genetic algorithm, and exponential adaptive genetic algorithm. The improved algorithm used in this paper is based on the linear adaptive genetic algorithm [6] proposed by Srinivas M, which is shown in Formula (2):

$$
\begin{aligned}
& P_{\mathrm{c}}=\left\{\begin{array}{l}
\frac{k_{1}\left(f_{\max }-f^{\prime}\right)}{f_{\max }-f_{\text {avg }}}, f^{\prime} \geq f_{\text {avg }} \\
k_{2}, f^{\prime}<f_{\text {avg }}
\end{array}\right. \\
& P_{\mathrm{m}}=\left\{\begin{array}{l}
\frac{k_{3}\left(f_{\max }-f\right)}{f_{\max }-f_{\text {avg }}}, f \geq f_{\text {avg }} \\
k_{4}, f<f_{\text {avg }}
\end{array}\right. \\
& f_{\max } \text {--The maximum fitness value of the individual in the current population } \\
& f^{\prime} \text {-Larger adaptive values of the two individuals to cross }
\end{aligned}
$$

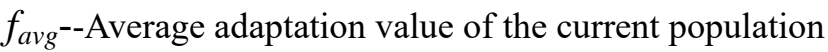

$$
\begin{aligned}
& f \text {--The adaptive value of the individual to be variant } \\
& P_{c} \text {-- crossover probability } \quad \mathrm{P}_{\mathrm{m}^{-}} \text {-mutation probability }
\end{aligned}
$$


When the individual fitness is large and close to the maximum value of the population fitness, $P_{c}$ and $\mathrm{P}_{\mathrm{m}}$ of the algorithm will be very small. When the individual adaptation value is the optimal value in the population, the variation probability is zero; no crossover and mutation operations are performed on the optimal individuals. When the level of the whole population fitness value is on average, it's feasible of Srinivas $\mathrm{M}$ taking $f_{\max }-f_{\text {ava }}$ as the standard to measure the population diversity. But when the adaptive value of the best individual in the population is an outlier, that is, the value is far from the sequence of the general level of maximum and minimum values, the use of $f_{\max }-f_{\text {ava }}$ to measure population diversity will lose its significance. In order to prevent this kind of situation, we use the standard deviation of individual fitness in the population to replace the $f_{\max }-f_{\text {ava }}$, and derive the adaptive mutation crossover probability[7], which is shown in Formula(3), and $P c_{1}=0.7, P c_{2}=0.1, P_{\mathrm{m} 1}=0.1, P_{\mathrm{m} 2}=0.001$ :

$$
\begin{aligned}
& P_{c}=\left\{\begin{array}{l}
P_{c 2}+\left(P_{c 1}-P_{c 2}\right) \arctan \left(\frac{f_{\max }-f^{\prime}}{\sqrt{\frac{1}{n} \sum_{i=1}^{n}\left(f_{i}-f_{\text {avg }}\right)^{2}}}\right) \frac{2}{\pi}, f^{\prime} \geq f_{\text {avg }} \\
P_{c 1}, f^{\prime}<f_{\text {avg }}
\end{array}\right. \\
& p_{m}=\left\{\begin{array}{l}
p_{m 2}+\left(p_{m 1}-p_{m 2}\right) \arctan \left(\frac{f_{\max }-f}{\sqrt{\frac{1}{n} \sum_{i=1}^{n}\left(f_{i}-f_{\text {avg }}\right)^{2}}}\right) \frac{2}{\pi}, f \geq f_{\text {avg }} \\
p_{m 1}, f<f_{\text {avg }}
\end{array}\right.
\end{aligned}
$$

In order to verify the performance of this improved adaptive genetic algorithm, this paper selected a test function that contains four local optimal solutions; The global optimal solution is often surrounded by the worst solution. If the population convergence of genetic algorithm is not good, it is very easy to fall into local optimum [10]. And it quotes linear adaptive genetic algorithm (LAGA) [6], cosine adaptive genetic algorithm (CAGA) [8], index (exponential) adaptive genetic algorithm (IAGA) [9] to compare the performance. The algorithm in this paper is called AAGA [7] by using the anti trigonometric function. The function description is shown in Table 2.

Table 2 test functions

\begin{tabular}{l|lc}
\hline test function & optimum point & optimum \\
\hline $\max f=\left(\frac{a}{b+\left(x_{1}^{2}+x_{2}^{2}\right)}\right) 2+\left(x_{1}{ }^{2}+x_{2}{ }^{2}\right)^{2}$, & $(0,0)$ & 3600 \\
$x 1, x 2 \in[-5.12,5.12], a=3, b=0.05$ & & \\
\hline
\end{tabular}

The parameters are set as shown in Tables 3 with the requirements of the parameters of these algorithms, the max evolution of the algorithm is 300 times. 
Table3 Genetic algorithm parameter table

\begin{tabular}{l|llll}
\hline parameter setting & $\mathrm{P}_{\mathrm{c} 1} \mathrm{P}_{\mathrm{c} 2}$ & $\mathrm{P}_{\mathrm{m} 1}$ & $\mathrm{P}_{\mathrm{m} 2}$ & \\
\hline LAGA & 0.9 & 0.6 & 0.1 & 0.001 \\
CAGA & 0.9 & 0.1 & 0.2 & 0.001 \\
IAGA & 0.8 & 0.6 & 0.04 & 0.005 \\
AAGA & 0.8 & 0.6 & 0.04 & 0.001 \\
\hline
\end{tabular}

The test function is run 100 times to obtain the convergence times, average convergence algebra and the average convergence value of the four algorithms, the results are shown in Table 4.

Table 4 Comparison of operating results of four algorithms

\begin{tabular}{|c|c|c|}
\hline convergence times & average convergence algebra & average convergence value \\
\hline LA CA IA AA & LA CA IA AA & LA CA IA AA \\
\hline GA GA GA GA & GA GA GA GA & GA GA GA GA \\
\hline $\begin{array}{llll}91 & 89 & 90 & 50\end{array}$ & $\begin{array}{llll}50 & 127 & 152 & 98\end{array}$ & 3389347635173591 \\
\hline
\end{tabular}

In terms of the average fitness, the population of this algorithm has more excellent individuals and relatively stronger adaptive ability than that of other adaptive genetic algorithms. The algorithm has certain advantages in both solving the maximum and minimum values; In terms of the optimal adaptation, the algorithm can achieve the optimal solution more quickly and has strong adaptive ability to run from local optimum to global optimum. And it has a good advantage in three aspects of the convergence times, average convergence algebra and the average convergence value.

\section{The solution of storage location allocation model}

The slotting optimization problem is actually a multi-objective problem; The multi-objective optimization problem is not optimal in the absolute sense, and can only balance the coordination of multiple objectives to achieve the Pareto optimal solution [11]. At present, there are many methods to solve the multi-objective problem by using the genetic algorithm, including the weight coefficient method, the coordinate selection method, and the permutation selection method and so on. This paper uses the parallel selection method of the genetic algorithm to solve the location allocation model. The solving process is mainly composed of 4 parts: coding mechanism, control parameters, fitness function and genetic operator [12], and the flow chart is shown in Figure 3:

(1)Encoding: use binary coding to encode chromosomes. Assumed that the warehouse has $16 * 32$ location and that the entire warehouse is divided into16 rows and 32 columns, each section using horizontal and vertical coordinates $(i, j)(i=1,2,3 \ldots .16 ; j=1,2,3 \ldots 32)$ calibration. Because the binary encoding method is used, horizontal coordinates using four bit binary coded 1111 representation, vertical coordinates using five bit binary coded 11111 representation, the location coordinates convert to mark (iiii, iiiii) $(\mathrm{i}=0,1)$ form. Because the genetic algorithm needs to do selection, cross and compiler operation in the two-dimensional space of location selection dynamically, this way is in low efficiency. So in this paper, we use the combination of horizontal 
and vertical coordinates as one coding (iiii, iiiii) $\rightarrow$ iiiiiiiii.

(2)Decoding process: the whole chromosome is divided into two parts according to the length of the binary string specified in the code, and then the horizontal and vertical coordinates of the binary string are transformed into the corresponding decimal to get specific location according to the decimal digits.

(3)The population initialization: crossover and mutation probability, $P_{\mathrm{c} 1}=0.8, P_{\mathrm{c} 2}=0.5, P_{\mathrm{m} 1}=0.04$, $P_{\mathrm{m} 2}=0.001$, population size 100 , max evolution times 3000 .

(4)Fitness function designation: the calculation of fitness value needs to use the data in Table 1 and the relevant data of the goods put into the warehouse, including goods order number, ID number, quantity of goods and the height of each package. After determining the above data, we use the five mathematical models which are established in the storage location assignment model to calculate the fitness value of the storage location, obtaining five fitness value matrixes of $16 * 32$, and then look up the table to find the fitness value of a location under a certain function in the genetic operation of the genetic algorithm.

(5)Genetic manipulation Use the improved algorithm to calculate the probability of crossover and mutation. The selection operator of the genetic algorithm uses the back type stochastic remainder algorithm, the crossover operator using the single point crossover algorithm, and the mutation operator using the discrete mutation algorithm. The location height limit enterprises provide is referred to and modified according to the actual situation when applied.

Table 5 some key unknown data in storage

\begin{tabular}{l|l}
\hline $\begin{array}{l}\text { variable } \\
\text { symbol }\end{array}$ & variable description \\
\hline order_flag $_{i i}$ & $\begin{array}{l}\text { Decision variable. order_flag } \\
\text { (i,j) coordinates has goods of the same order; order_flag } \\
\text { indicates that location with }(\mathrm{i}, \mathrm{j}) \text { coordinates not has goods of } \\
\text { the same order } \\
\text { kind_flag } \\
\text { Decision variable. kind_flag } \\
\text { (i,j) coordinates has the same kind of goods ; kind_flag } \\
\text { indicates that location with }(\mathrm{i}, \mathrm{j}) \text { coordinates not has the same } \\
\text { kind of goods }\end{array}$ \\
\hline
\end{tabular}

Some of the key unknown data is listed in Table 5. The XY coordinate and the maximum XY coordinates in the warehouse are the constant values, which obtained from the actual planning effect of each warehouse.

The following figures show the simulation process of the storage location assignment model using AAGA to solve the optimal solution. Figure 1 shows the initial state of the warehouse. The square represents that the location is higher than $250 \mathrm{~cm}$ and cannot place goods anymore; The diamonds represent the height of the space is between [100,250); The delta represents the height of the space is between $[50,100)$; The upper triangle represents the height of the space is between $[0$, 50). In Figure 2 the star- mark coordinates $(8,18)$ represents the recommended optimal goods location. 


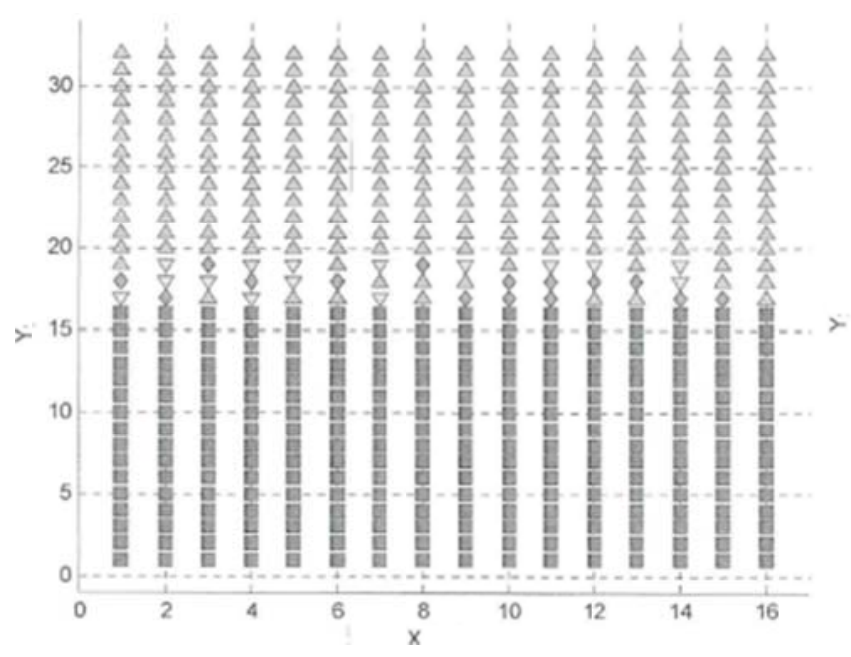

Figure 1 Initial state warehouse

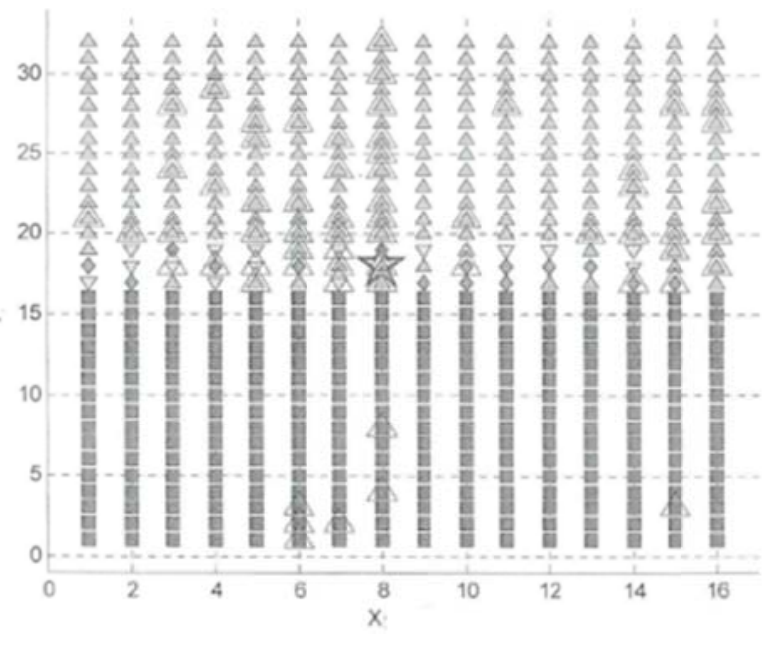

Figure 2 A warehouse marked by genetic algorithms

In the storage system, referring to the storage location assignment model, the improved adaptive genetic algorithm can be realized by java and other advanced languages. When doing the storage operation, the warehouse keepers scan the two-dimensional code attached to the goods to get information about the goods, enter the number inbound, and submit the information to the server through the android client. The server carries out the genetic operations according to the parameters and algorithm of the transmission, then the recommended storage location is returned to the client on display.

\section{Conclusions}

In this paper, a new adaptive genetic algorithm is proposed to improve the representation of the population diversity in the adaptive function. Compared with other adaptive genetic algorithms, this algorithm has certain advantages in the average fitness and optimal fitness. Secondly, this paper analyzes the factors affecting the warehousing operations according to the basic principle of warehousing and inventory status, summarizes the storage location selection as a problem of the multi-objective optimization, and sets up the mathematical model. Finally, the parallel selection method of genetic algorithm is used to solve the storage optimization model and the multi-objective optimization, the process of which includes encoding、 decoding、 data initialization and fitness function design. The results show that using this improved adaptive genetic algorithm in building the storage optimization model can better solve the problem of storage and choose the best location.

\section{References}

[1]Shenglong Zuo, Jun Liu. Modern warehousing operations management [M].Beijing: China Materials Press, 2006:161-162.

[2]Yongjie Ma, Zhaoyuan Jiang, Zhiming Yang. Dynamic storage allocation of automated warehouse based on genetic algorithm [J].Journal of Southwest Jiao Tong University, 2008, 3(43):415-420.

[3]Yunwei Shang, Yuhuang Qiu,Changyou Liu. Automated warehouse location assignment 
optimization problem research [J].Computer engineering and Application. 2004(26):16-17.

[4]Lingying Zheng, Xin Zhang, Yonghua Yan. Design of warehousing slotting optimization system in Logistics Center [J].Logistics technology.2006 (06):33-34.

[5]Shiwen Tai.Research on the optimization of logistics center facility layout of auto parts [D].Dalian: Dalian University of Technology, 2013.

[6]Srinivas M, Patnaik L M. Adaptive probabilities of crossover and mutation in genetic Algorithms [J], Systems, Man and Cybernetics, IEEE Transactions on, 994, 24(4):656-667.

[7]Zhilong Yang. Research and implementation of warehouse management system based on genetic algorithm [D].Qingdao: Ocean University of China, 2014.

[8]Shan Shi,Qinyun Li,Xinhua Wang.Optimal design of Brushless DC motor based on adaptive genetic algorithm [J].Journal of Xi'an Jiaotong University,2002(12):1215-1218.

[9] Jing Jin, Yong Su. An improved adaptive genetic algorithm [J]. Computer engineering and Applications, 2005(18):64-69.

[10]Ruifeng Han.Principle and application of genetic algorithm [M].BeiJing: Weapon Industry Press, 2010:89-90.

[11]Carlos F M, Peter F J. An overview of evolutionary algorithms in multi objective optimization [J]. Evolutionary Computation, 1995, 3(1):1- 16.

[12]Xiongzhi Wang. Distribution center order picking method study [M].Beijing: China Economic Publishing House, 2008:125. 